\title{
Essais
}

Revue interdisciplinaire d'Humanités

14 | 2018

Plurilinguismes en construction

\section{Mario Sibilio, La Didattica Semplessa}

\section{Mario Pasquariello}

\section{OpenEdition}

\section{Journals}

Edizione digitale

URL: http://journals.openedition.org/essais/401

DOI: 10.4000/essais.401

ISSN: 2276-0970

\section{Editore}

École doctorale Montaigne Humanités

\section{Edizione cartacea}

Data di pubblicazione: 1 giugno 2018

Paginazione: 201-207

ISBN: 979-10-97024-06-2

ISSN: 2417-4211

Notizia bibliografica digitale

Mario Pasquariello, «Mario Sibilio, La Didattica Semplessa », Essais [En ligne], 14 | 2018, mis en ligne le 01 décembre 2019, consulté le 23 septembre 2020. URL : http://journals.openedition.org/essais/401 DOI : https://doi.org/10.4000/essais.401 
Mario Sibilio, La Didattica Semplessa, Napoli, Liguori Editore, 2014.

In La Didattica Semplessa Maurizio Sibilio, docente di "Didattica e Pedagogia speciale" dell'Università di Salerno, propone una visione semplessa della didattica. Elaborato dal fisiologo francese Alain Berthoz nel 2011, cui si deve anche la prefazione al volume, il concetto di semplessità si fonda sull'assunto che le soluzioni elaborate dagli organismi viventi per decifrare e fronteggiare la complessità possano essere considerate valide ed applicabili all'intera classe dei sistemi complessi adattivi. L' analogia operata da Sibilio tra sistema didattico e sistema vivente sollecita la riflessione sulla possibile estensione al discorso sulla formazione e sull'educazione dei principi e delle proprietà che Berthoz attribuisce agli organismi viventi.

Sibilio propone una visione del sistema didattico come "sistema complesso adattivo" e nella sua dettagliata argomentazione sostiene una visione di sistema la cui struttura "reticolare" è costituita da unità composite che sono, a loro volta, sistemi complessi adattivi con legami ed interazioni di tipo non lineare. Questa analogia gli consente di creare le condizioni per costruire un modello di descrizione del fenomeno didattico. Le suggestioni semplesse risolvono la questione relativa alla dimensione fattuale e operativa di una nuova literacy metodologico-didattica orientata a leggere coscientemente le scelte metodologiche e a ripensare le strategie didattiche sulla base dei meccanismi di azione. In particolare, la proiezione semplessa del fenomeno didattico induce a spostare il locus del controllo del sistema sull'atto, quale forma "incarnata" e "situata" della cognizione, in cui si realizza una sintesi di percezione e azione non priva di ricadute sul processo di insegnamento-apprendimento.

La rinnovata prospettiva visione del rapporto tra percezione, azione e cognizione che ne consegue contribuisce, tramite una specifico studio sui significati e sulle funzioni dell'atto in campo didattico, alla comprensione della complessità del fenomeno didattico. L'atto, in quanto rivolto ad uno scopo e sostenuto da un'intenzione, consente di organizzare sia la percezione sia il mondo percepito, per orientare il processo di insegnamento-apprendimento verso finalità educative. L'atto -come Sibilio aveva già sostenuto nel 2012- è, nel contempo, espressione di decisioni riconducibili tanto ai saperi della cultura di appartenenza, quanto ai personali modelli di interpretazione che si integrano, nella dimensione didattica, con i modelli pedagogici e didattici impliciti.

Ciò rafforza l'idea di una transdisciplinarità percorribile tra biologia, pedagogia e didattica nel riconoscimento di una sintesi coerente di nature e nurture che definitivamente si risolve nell'atto, quale fondamento del senso educativo e didattico. Il lavoro di ricerca di Alain Berthoz ha avuto il merito di attivare la riflessione sul riscontro, nel sistema didattico, di proprietà e principi che regolano il processo di adattamento dei soggetti che determinano 
con il proprio agire i processi di insegnamento-apprendimento in una dimensione autopoietica della formazione. In un sistema didattico che si caratterizza per complessità e naturale tendenza all'adattamento, le proprietà didattiche semplesse possono essere interpretate come modalità di interazione, ovvero schemi delle relazioni che si generano tra le componenti del sistema didattico a loro volta regolati da principi che influenzano la specifica organizzazione. Le proprietà costituiscono, in questo senso, i presupposti funzionali dell'azione didattica nella sua interazione con l'ambiente e, conseguentemente, i principi semplessi dell'azione didattica rappresentano regole semplici sulle quali si fondano i modelli adattivi dell'agire didattico che consentono di decifrare e fronteggiare la complessità formativa. Riconoscendo questa assoluta centralità dell'azione, gli studi sulla possibile declinazione della semplessità in didattica riportano necessariamente ad una riflessione più ampia sulla formazione.

Arginati i rischi di una deriva esclusivamente descrittiva della complessità, una lettura semplessa consente di individuare principi trasferibili in diversi campi d'azione, la cui consapevolezza aiuta ad orientare anche il processo formativo verso finalità educative. Dallo studio di specifiche modalità interattive derivanti da schemi funzionali all'adattamento, emerge una possibile impostazione dell'azione didattica che implica meccanismi decisionali a livello cosciente e subcosciente, che si giocano in uno spazio-tempo molto ridotto, ma protraggono i propri effetti in dinamiche di sviluppo. In questo senso la semplessità consente di riconsiderare i possibili significati del processo formativo e le sue implicazioni nella conquista dell'autonomia personale, sollecitata costantemente dai sillabi e dalle diverse indicazioni ministeriali, fulcro delle istituzioni educative e presupposto per la piena affermazione del soggetto e per l'esercizio dei suoi diritti di cittadino. Nell'immaginario comune sono la scuola e l'università a dover rimuovere gli ostacoli alla piena affermazione della persona, mentre in una logica sistemica è la capacità di adattamento del soggetto a consentire il superamento della complessità della vita e il districarsi nelle diverse situazioni problematiche che caratterizzano le diverse esperienze.

Se da un lato l'evoluzione della conoscenza e l'inarrestabile progresso tecnologico sembrano offrire la risoluzione a molte delle complessità della vita quotidiana, dall'altro creano ulteriori livelli di complessità, che a loro volta richiedono da parte dell'individuo un costante processo di adattamento e la messa a punto di soluzioni sempre più raffinate. La rapidità e l'efficacia nell'operare in diverse situazioni problematiche; la flessibilità e la capacità di accogliere il cambiamento; la capitalizzazione delle esperienze pregresse per prevedere gli effetti e prevenire le conseguenze delle proprie azioni; il ricorso a schemi codificati a livello generale nelle diverse situazioni problematiche sono indispensabili in tutti i campi dell'esperienza. Si tratta di strategie di adattamento potenzialmente trasferibili a tutti gli ambiti dell'attività umana, che rappresentano un patrimonio adattivo di tutti gli esseri. Di fatto è lo sviluppo di capacità metacognitive che porta a riflettere e sviluppare conoscenza sulle abilità richieste per fronteggiare la complessità e non esserne sopraffatti. 
In questa prospettiva, le istituzioni educative e formative, pur nel rispetto delle proprie prerogative istituzionali e senza sottovalutare la complessità del processo formativo, dovrebbero promuovere interventi formativi finalizzati a costruire e/o sviluppare in azione capacità e competenze per fronteggiare la complessità.

L'autore porta quali argomenti a favore della propria tesi una rilettura delle Indicazioni nazionali per il curricolo della scuola dell'Infanzia e del primo ciclo di istruzione del 2012 in chiave semplessa. Il Regolamento è il contributo della scuola italiana all'allineamento con le Raccomandazioni del Parlamento Europeo e del Consiglio d'Europa del 2006 e recepisce gli indirizzi relativi alla necessaria costruzione di specifiche competenze trasferibili nei diversi contesti di vita cercando di capitalizzare i risultati della formazione scolastica nelle possibili situazioni problematiche e favorire un processo di autonomia adeguato al grado di complessità della vita nel XXI secolo.

Sibilio rileva nel documento europeo che individua le competenze chiave un orizzonte alquanto complesso che si esplica nell'aspettativa che le condizioni indispensabili al superamento di situazioni problematiche si creino attraverso lo svolgimento dell'esperienza formativa. Altrettanto complesso è il profilo sistemico e la visione della scuola e del processo di insegnamentoapprendimento che emana dalle Indicazioni nazionali.

Gli aspetti della complessità del sistema didattico italiano sono reperiti anche nell'affermazione dell'Autonomia scolastica. La legge 59 del 1997 e il successivo Decreto del Presidente della Repubblica n 275 del 1999, annovera tra le varie sue finalità quella di liberare le enormi potenzialità dell'agire didattico attraverso un'autonomia amministrativa, didattica e organizzativa della scuola, che ridetermina le relazioni e le interazioni all'interno di un sistema complesso adattivo come quello educativo-didattico.

Gli sforzi compiuti dalla ricerca educativa per ridefinire la rete di vincoli, propositi, professionalità, attori, fini, condizioni, materiali, tendenze centralizzatrici e spinte locali del sistema educativo, non hanno tuttavia prodotto una fattiva progettualità didattica, spesso limitandosi alla mera descrizione della complessità del sistema. L'autonomia scolastica avrebbe dovuto rappresentare una preziosa occasione per riflettere sulle reali possibilità e sulle eventuali modalità di innovazione culturale, strutturale e funzionale delle istituzioni scolastiche e della didattica, rispondendo ai principi normativi ed utilizzando proprietà adattive di tipo sistemico, in grado di favorire e promuovere l'esperienza formativa.

La coesistenza tra autonomia didattica e libertà di insegnamento del docente, da un lato, e obiettivi e indicazioni definiti centralmente dalla struttura ministeriale, dall'altro, rende particolarmente complessa l'azione didattica. Il dettato normativo riconosce una rinnovata responsabilità della 
professione docente, la quale implica un modello di formazione professionale dell'insegnante, traducibile in percorsi formativi per l'acquisizione di sofisticate capacità che aiutino ad identificare soluzioni didattiche efficaci.

Tuttavia le disposizioni sull'autonomia, secondo Sibilio, non si sono dimostrate in grado di "liberare la didattica" e realizzare l'auspicata circolarità prassi-teoria-prassi nella promozione degli apprendimenti. È mancata quella promozione di adeguate competenze professionali dei docenti, grazie alla quale la legge avrebbe potuto agevolare lo sviluppo dei sistemi di qualità dell'istruzione e della formazione, attribuendo nuove proprietà di carattere sistemico che avrebbero garantito una costante funzione adattiva della didattica e una ricaduta positiva sulle relazioni tra insegnamento-apprendimento in una logica competence-based.

Nel campo della formazione docente l'adozione del concetto di semplessità, la trasferibilità e l'esercizio dei suoi principi potrebbero costituire un'originale opportunità per riconsiderare i presupposti e le modalità delle competenze didattiche del docente.

Uno studio pilota finalizzato a rilevare in che modo gli insegnanti fronteggiano la sfida della complessità del processo di insegnamento-apprendimento, condotto dall'équipe del Prof. Sibilio, ha fatto emergere il grado di consapevolezza nell'agire didattico. L'indagine si è proposta di esplorare quanto le convinzioni dei docenti relative al processo di apprendimento aderissero a modelli teorici e a metodi e strumenti sviluppati nell'ambito di tali modelli, e quanto questa adesione fosse consapevole ed esplicita. L'obiettivo era di individuare un lessico comune ai docenti coinvolti sulla natura, sulle caratteristiche e sulle funzioni del fenomeno e dell'azione didattica; il tipo di legame che il docente ritiene di stabilire nel processo di insegnamento-apprendimento; $\mathrm{i}$ presupposti teorici e i meccanismi che sottendono all'interazione del processo di insegnamento-apprendimento; gli atteggiamenti professionali prevalenti finalizzati a consentire l'auto-organizzazione dell'azione didattica dei docenti coinvolti. Con queste premesse, l'indagine si è inserita funzionalmente nel filone di ricerca sulla semplessità in didattica e ha cercato di stabilire a) in che termini la formazione docente o l'esperienza sul campo costituissero risorse epistemologiche capaci di attribuire all'azione didattica proprietà semplesse; b) se l'attività di insegnamento si riferisse coscientemente a specifici principi o regole da cui derivare modelli didattici.

Il ricorso a un approccio quali-quantitativo tramite somministrazione di un questionario ha consentito di effettuare un'indagine sulle conoscenze e sulle concezioni personali di un gruppo di docenti circa il processo di insegnamento-apprendimento. Sono stati raggiunti 615 docenti, distribuiti nel territorio della Campania e provenienti da ogni ordine e grado di scuola. Dai risultati è stato possibile identificare in che misura l'epistemologia personale dei docenti fosse riconducibile ad un quadro teorico consapevole ed in che 
termini questo influenza, in ambito didattico, le scelte decisionali e l'impostazione didattica. Il docente sembra costruire una personale rete di risorse epistemologiche e di buone pratiche più o meno consapevole ed orientata da principi guida. Gli esiti dello studio pilota sembrano recepire le sollecitazioni derivanti dalla proposta scientifica di Berthoz nell'interpretare l'esigenza di riconoscere proprietà e principi regolativi in grado di fronteggiare le complessità dell'esperienza formativa. Lo studio sembra, infatti, giustificare un esplicito parallelo tra l'agire del docente, che seleziona e mette in campo strategie volte a conseguire obiettivi formativi in contesti la cui complessità non è determinabile a monte, e l'agire dell'organismo, che affronta la complessità semplicemente attraverso l'azione. L'agire didattico risulta caratterizzato da una riduzione significativa di qualsiasi complessità precostituita e dall'introduzione di una complessità accessoria, relativa alla situazione locale, rispondendo al principio semplesso della deviazione. Le continue deviazioni che il docente opera, nel tentativo di semplificare, generano ulteriore complessità.

La semplessità in questo senso, come insieme di principi semplificativi, appare una pista decisamente promettente, non soltanto come chiave interpretativa del fenomeno didattico, ma soprattutto come linea guida per la formazione docente, in grado di recepire e soddisfare, a livello di sistema, la necessità di risolvere complessità alla base dell'intervento didattico.

Per gettare le fondamenta teoriche di una didattica semplessa, Il saggio offre altresì delle piste per una necessaria verifica di applicabilità dei principi semplessi alla ricerca e alla pratica didattica e, nel contempo ridefinire confini e modalità per una formazione docente in grado di costruire competenze atte a fronteggiare la complessità formativa.

Nelle pagine di Sibilio si illustrano a grandi linee alcuni progetti, elaborati e sperimentati dal gruppo di ricerca didattica dell'Università degli Studi di Salerno in collaborazione con l'Università Suor Orsola Benincasa di Napoli in diversi ambiti della ricerca didattica, che condividono l'orizzonte teorico della semplessità in didattica e ne rappresentano una prima applicazione sperimentale. I progetti descritti si propongono di indagare sulla fruibilità didattica della semplessità, attraverso specifiche ricerche traducibili in sperimentazioni di dimensione nazionale ed internazionale. I percorsi di ricerca descritti sono finalizzati, inoltre, a riconsiderare il significato della formazione docente in campo didattico, ancorata in molti casi in Italia a modelli lineari tra teoria e prassi, oggi inadeguati a rispondere alla complessità del processo di insegnamento-apprendimento.

1. Il progetto "Corpo e movimento per una didattica semplessa" evidenzia la dimensione semplessa nell' uso del corpo in movimento, in particolare del gesto, nel processo di insegnamento-apprendimento che costituisce, sul piano metodologico, una deviazione per il fronteggiamento della complessità formativa, al fine di integrare e ampliare l'agire didattico dei docenti. 
Nello specifico, una metodologia centrata sul corpo in azione può: a) affiancare le modalità di insegnamento già in uso da parte dei docenti; b) integrare e ampliare l'agire didattico; c) rappresentare una modalità alternativa alla propria metodologia di insegnamento.

2. Obiettivo del progetto "La semplessità del Transmedia Digital Storytelling per promuovere l'apprendimento della lingua inglese negli studenti dislessici" è suggerire eventuali applicazioni semplesse alla pedagogia e alla didattica speciale, nella prospettiva inclusiva adottata dalla scuola italiana. Il focus del progetto è l'utilizzo delle potenzialità del transmedia digital storytelling all'interno di una possibile metodologia didattica semplessa per facilitare l'apprendimento della lingua inglese in soggetti con bisogni educativi speciali. Il progetto si concentra, in particolare, sulla manipolazione dello spazio "percepito, vissuto e concepito" per sostenere la concettualizzazione (che è la base dell'elaborazione linguistica) e sulle modalità attraverso le quali è possibile spazializzare gli stessi concetti attraverso media differenti.

3. Il progetto "Acchiappanuvole" mira invece a progettare e sviluppare un exergame che faciliti lo sviluppo della lateralizzazione, nel contesto dei disturbi specifici di apprendimento legati all'elaborazione visuo-spaziale e al deficit motorio-percettivo. L'attuale stato del progetto ha portato alla creazione di un exergame, basato sulla tecnologia Microsoft Kinect, in cui il giocatore controlla i movimenti di un avatar sullo schermo. Il target di riferimento sono i bambini che frequentano la scuola primaria.

4. Il focus del progetto "Spazialità didattiche: misurare l'empatia nel processo di insegnamento-apprendimento" è il rapporto tra empatia e Game-Based Learning, al fine di individuare parametri utlili a misurare l'empathetic embodiment. Seguendo l'approccio fenomenologico, l'empatia è un meccanismo molto più complesso di simpatia, perché richiede un cambiamento di prospettiva e una qualche forma di "out of body experience" per separare noi stessi mentalmente dal nostro corpo e viaggiare nel corpo altrui. Il progetto presenta le linee guida alla base della concezione e dello sviluppo di un gioco narrativo progettato con lo scopo di misurare la capacità di assumere una diversa prospettiva.

In definitiva La didattica semplessa auspica percorsi formativi che esercitino a selezionare tra le azioni possibili quelle maggiormente idonee ad affrontare le diverse situazioni problematiche. Sul piano metodologico-didattico, Sibilio immagina l'istituzione di laboratori semplessi della meta-cognizione attorno all'utilizzo di principi di semplificazione trasferibili ai diversi contesti. La conquista dell'autonomia personale, evocata dalle Indicazioni nazionali e dai curricula scolastici, si risolverebbe dunque nella capacità di riconoscere l'importanza di principi semplessi come: rifiutare ed inibire; specializzare e selezionare; anticipare e prevedere in ragione della propria esperienza; deviare da una soluzione per scegliere un'alternativa; cooperare ed utilizzare le medesime strategie risolutive in situazioni differenziate. 
Inoltre la semplessità ripropone un'idea di formazione fondata sul monitoraggio e l'autoregolazione di semplici regole di adattamento messe a punto dall'evoluzione. Una volta riconosciuta l'importanza di queste strategie metacognitive, scuola e università dovrebbero promuovere metodologie didattiche in grado di costruire, sviluppare ed applicare queste capacità di fronteggiamento della complessità attraverso esperienze formative grazie alle quali possano emergere e tradursi in azione schemi adattivi semplificatori.

Mario Pasquariello

Università Cattolica del Sacro Cuore Milano Université Bordeaux Montaigne mario.pasquariello@unicatt.it 